\title{
On the Extended Relationships Among EVM, BER and SNR as Performance Metrics
}

\author{
Rishad Ahmed Shafik \\ Islamic University of Technology \\ Gazipur 1704, Bangladesh \\ rishad09@gmail.com
}

\author{
Md. Shahriar Rahman \\ Islamic University of Technology \\ Gazipur 1704, Bangladesh \\ shahriar@iut-dhaka.edu
}

\author{
AHM Razibul Islam \\ Kyung-Hee University \\ South Korea \\ razib3002@gmail.com
}

\begin{abstract}
In this paper, we relate the error vector magnitude (EVM) bit error rate (BER) and signal to noise ratio (SNR). We also present the fact that with such relationship it would be possible to predict or in cases substitute EVM in places of BER or even SNR. In doing so, we first define EVM with normalization so that the definition stands for multi-modulation systems, viz. binary phas shift keying (BPSK), quadrature phase shift keying (QPSK) etc. We also compare among the different performance metrics and show that EVM can be equivalently useful as signal to noise ratio and bit error rate. The relationships are based on stream based communication systems. A few Monte Carlo simulations are carried out to illustrate the performance of EVM based on these relationships.
\end{abstract}

\section{INTRODUCTION}

Signal to noise ratio (SNR), error vector magnitude (EVM) and bit error rate are common performance metrics for assessing the quality of communication [7]. SNR is a direct measure of the relative power of the noise compared to signal. Since noise is source of all errors for simplistic Gaussian noise channel model, SNR can be used to predict the performance of the system in terms of correctness of the reception. The later can be measured using the bit error rate (BER), which gives a simple one-to-one binary decision as to whether a bit is erroneous or not. Due to the simplicity of comparison, BER has been a major choice to engineers, industries and researchers[6], [5], [7]. BER performance against SNR is the popular performance criterion that is used in today's communications systems. On the other hand, EVM is a measure of errors between the measured symbols and expected symbols. The use of EVM as a performance metric is limited to radio frequency engineering to infer reception the performance at the receiver earlier than the BER. In this paper, we show the relationship among BER, SNR and EVM extended from [7] and [2]. However, the relationships are based on assumption that the communication is stream based or the size of the packet is such that the number of samples is much higher than the number of distinct modulation symbols.

The relationships among these metrics are vital to allow re-thinking on using these parameters towards making adaptive systems. As an example, for BER measurement, it is incumbent that signal must be demodulated first at the receiver side. For many of todays adaptive systems, viz. minimum bit error rate (MBER) based adaptive modulation systems etc., this means that for every update in the adaptive algorithm, it has to receive feedback from the receiver end[8]. Since EVM can give the desired performance metric before the demodulation can actually takes place, often BER calculations can be avoided for large packets. This can be done without any major change of algorithm in adaptive systems, since BER is a direct consequence of EVM. But again, due to complex mathematical operations that needs to be done in the digital signal processor for calculation of EVM, for performance measures for systems with small packet communication BER versus SNR can be easier and more useful. In Section III and IV, BER and EVM will be separately derived from SNR and later EVM definition would be presented in terms of BER. In VI, theoretical and empirical observations would be shown through simulations showing the relation of EVM with BER. Finally, in Section VII, we extend discussions on these results and show future directions for such performance metric.

\section{Signal to Noise Ratio}

Signal to noise ratio is a relative measure of the signal power compared to the noise power. Assuming Gaussian noise model for wireless channels and complex signals, SNR can be defined as

$$
\begin{aligned}
S N R & =\frac{\text { Signal Power }}{\text { Noise Power }} \\
& =\left[\frac{\frac{1}{T} \sum_{t=1}^{T}\left[\left(I_{t}\right)^{2}+\left(Q_{t}\right)^{2}\right]}{\frac{1}{T} \sum_{t=1}^{T}\left|n_{I, t}\right|^{2}+\left|n_{Q, t}\right|^{2}}\right]
\end{aligned}
$$

Here $I_{t}$ and $Q_{t}$ are the in-phase and quadrature signal amplitudes of the $M$-ary modulations, $n_{I, t}$ and $n_{Q, t}$ are the in-phase and quadrature noise amplitudes of the complex noise being considered. Equation 1 shows a direct measure of SNR, which can be used in Monte Carlo simulation procedures for large symbol streams, such that $T>N$, where $N$ is the number of unique modulation symbols. Often these estimates are simplified by considering the measure of the ratio of variances of signal and the noise, when both are zero mean processes. For systems, which are sampled at data rate, $\frac{E_{s}}{N_{0}}$ gives the signal to noise ration directly, where $E_{s}$ is the symbol energy and $\frac{N_{0}}{2}$ gives the noise power spectral density. It is to be noted that $E_{s}=\log _{2} M E b$, for such systems. 


\section{BIT ERROR RATE}

Bit Error Rate (BER) is a commonly used performance metric which describes the probability of error in terms of number of erroneous bits per bit transmitted. BER is a direct effect of channel noise for Gaussian noise channel models. For fading channels, BER performance of any communication system is worse and can be directly related to that of the Gaussian noise channel performance[4]. Considering $M$-ary modulation with coherent detection in Gaussian noise channel and perfect recovery of the carrier frequency and phase, it can be shown that[4]

$$
P_{\mathrm{b}}=\frac{2\left(1-\frac{1}{L}\right)}{\log _{2} L} Q\left[\sqrt{\left[\frac{3 \log _{2} L}{L^{2}-1}\right] \frac{2 E_{b}}{N_{0}}}\right],
$$

where $\mathrm{L}$ is the number of levels in each dimension of the $M$-ary modulation system, $E_{b}$ is the energy per bit and $N_{0} / 2$ is the noise power spectral density. $Q[$.$] is the Gaussian co-error$ function and is given by[3]

$$
Q(x)=\int_{x}^{\infty} \frac{1}{\sqrt{2 \pi}} e^{\frac{-y^{2}}{2}} d y .
$$

Assuming raised cosine pulses with sampling at data rate, Equation 2 also gives the bit error rate in terms of signal to noise ratio as

$$
P_{\mathrm{b}}=\frac{2\left(1-\frac{1}{L}\right)}{\log _{2} L} Q\left[\sqrt{\left[\frac{3 \log _{2} L}{L^{2}-1}\right]\left[\frac{2 E_{s}}{N_{0} \log _{2} M}\right]},\right.
$$

where $\frac{E_{s}}{N_{0}}$ is the signal to noise ratio for the $M$-ary modulation system (as shown before) and raised cosine pulse shaping at data rate. Equation 4 defines the BER performance in terms on SNR.

\section{ERror Vector Magnitude}

Error Vector Magnitude (EVM) measurements are often performed on vector signal analyzers (VSAs), real-time analyzers or other instruments that capture a time record and internally perform a Fast Fourier Transform (FFT) to enable frequency domain analysis. Signals are down converted before EVM calculations are made[2]. With the insurgence of high speed communication requirements, highly efficient multiplexing systems like orthogonal frequency division multiplexing (OFDM) as specified in [6] is becoming the hinge of future communication systems. OFDM is now being used in different carrier standards in Wireless Local Area Networks (WLANs) and also being considered as a potential fourth generation communication system. The IEEE802.11a-1999 specification describes a set of different schemes that are used in adaptive fashion: binary phase shift keying (BPSK), 4 quadrature amplitude modulation (4-QAM), 16 quadrature amplitude modulation (16-QAM), 64 quadrature amplitude modulation (64-QAM) etc. The fact that pilots and training bits are always BPSK modulated, it is also possible to have more than one modulation scheme within a burst[7]. This requires that these modulation schemes are normalized to facilitate the calculation of EVM.

The normalization is derived such that the mean square amplitude of all possible symbols in the constellation of any modulation scheme is one. Thus, EVM is defined as the root-mean-square (RMS) value of the difference between a collection of measured symbols and ideal symbols. These differences are averaged over a given, typically large number of symbols and are often shown as a percent of the average power per symbols of the constellation. As such EVM can be mathematically given as[1]

$$
E V M_{\mathrm{RMS}}=\frac{\frac{1}{N} \sum_{n=1}^{N}\left|S_{n}-S_{0, n}\right|^{2}}{\frac{1}{N} \sum_{n=1}^{N}\left|S_{0, n}\right|^{2}},
$$

where $S_{n}$ is the normalized $n$th symbol in the stream of measured symbols, $S_{0, n}$ is the ideal normalized constellation point of the $n$th symbol and $N$ is the number of unique symbols in the constellation. The expression in Equation 5 cannot be replaced by their unnormalized value since the normalization constant for the measured constellation and the ideal constellation are not the same. The normalization scaling factor for ideal symbols is given by[7]

$$
|A|=\sqrt{\frac{1}{\frac{P_{v}}{T}}}=\sqrt{\frac{T}{P_{v}}},
$$

where $P_{v}$ is the total power of the measured constellation of $T$ symbols. For RMS voltage levels of in-phase and quadrature components, $V_{I}$ and $V_{Q}$ and for $T>N$, it can be shown that $P_{v}$ can be expressed as

$$
P_{v}=\sum_{t=1}^{T}\left[\left(V_{I, t}\right)^{2}+\left(V_{Q, t}\right)^{2}\right] \quad(W)
$$

The normalization factor for ideal case can be directly measured from $N$ unique ideal constellation points and is given by

$$
\left|A_{0}\right|=\sqrt{\frac{N}{\sum_{n=1}^{N}\left[\left(V_{I_{0, n}}\right)^{2}+\left(V_{Q_{0, n}}\right)^{2}\right]}} .
$$

Hence Equation 5 can be further extended using normalization factors in Equations 6 and 8 as

$$
E V M_{\mathrm{RMS}}=\left[\frac{\frac{1}{T} \sum_{t=1}^{T}\left|I_{t}-I_{0, t}\right|^{2}+\left|Q_{t}-Q_{0, t}\right|^{2}}{\frac{1}{N} \sum_{n=1}^{N}\left[\left(I_{0, n}\right)^{2}+\left(Q_{0, n}\right)^{2}\right]}\right]^{\frac{1}{2}},
$$

where $I_{t}=\left(V_{I_{t}}\right)|A|$ is the normalized in-phase voltage for measured symbols and $I_{0, t}=\left(V_{I_{0, t}}\right)\left|A_{0}\right|$ is the normalized in-phase voltage for ideal symbols in the constellation, $Q_{t}=$ $\left(V_{Q_{t}}\right)|A|$ is the normalized quadrature voltage for measured symbols and $Q_{0, t}=\left(V_{Q_{0, t}}\right)\left|A_{0}\right|$ is the normalized quadrature voltage for ideal symbols in the constellation. This is the definition which is now being used as the standard definition of the EVM in IEEE $802.11 a-1999^{T M}$ [6], [5]. 


\section{RELATIONSHIP AMONG EVM, BER AND SNR}

From Equation 9, it is evident that EVM is essentially the normalized error magnitude between the measured constellation and the ideal constellation. For Gaussian noise model, Equation 9 can be simplified in terms of noise in-phase component, $n_{I, t}$ and quadrature component, $n_{Q, t}$ as

$$
E V M_{\mathrm{RMS}}=\left[\frac{\frac{1}{T} \sum_{t=1}^{T}\left[\left|n_{I, t}\right|^{2}+\left|n_{Q, t}\right|^{2}\right]}{P_{0}}\right]^{\frac{1}{2}},
$$

where $P_{0}$ is the power of the normalized ideal constellation or the transmitted constellation. The numerator of Equation 10 sets up the normalized noise power. However, for $T>>N$, the ratio of normalized noise power to the normalized power of ideal constellation can be replaced by their unnormalized quantities, i.e. the Equation 10 rewritten as

$$
E V M_{\mathrm{RMS}} \approx\left[\frac{1}{S N R}\right]^{\frac{1}{2}}=\left[\frac{N_{0}}{E_{s}}\right]^{\frac{1}{2}} .
$$

In order to establish relationship between BER and EVM, SNR in Equation 11 can be expressed in terms of EVM as

$$
S N R \approx \frac{1}{E V M^{2}}
$$

Combining Equations 12 and 4, we can now relate the bit error rate directly with the error vector magnitude as follows

$$
P_{\mathrm{b}} \approx \frac{2\left(1-\frac{1}{L}\right)}{\log _{2} L} Q\left[\sqrt{\left.\left[\frac{3 \log _{2} L}{L^{2}-1}\right] \frac{2}{E V M_{\mathrm{RMS}}^{2} \log _{2} M}\right]},\right.
$$

\section{Simulations}

Few simulations have been carried out to illustrate the relation established in Equation 13. The bits are gray coded and then $M$-ary modulated, where $M=2$ for BPSK, $M=4$ for 4-QAM, $M=16$ for 16-QAM and $M=64$ for 64-QAM. Monte Carlo Simulation techniques are carried out using $10^{6}$ packets, each with the size of 1024 bits. The channel model used is Gaussian noise model. The normalization factors will be equal as shown in Equation IV since $1024>>M$ for any $M$-ary modulation scheme. The simulation results for BPSK, 4-QAM, 16-QAM and 64-QAM are presented in figures 1, 2 and 3 , respectively.

Figure 1 shows the BER versus SNR performance of different modulation systems. Note that due to the relationship set up between BER and EVM in Equation 13, the BER versus EVM curve shown in Figure. 2 shows the inverse relationship that exists between BER and EVM (with power term in log scale shown).

In order to also establish the fact that the normalized EVM is same for all modulation schemes, we have also carried out another simulation as shown in Figure 3. Due to normalization, the power levels for all different $M$-ary modulations are same and hence shows a one-to-one relationship between them.
In Figure 2, we note that there is a constant $2.89 \mathrm{~dB}$ difference between BPSK and 4-QAM, whereas there is a $6.85 \mathrm{~dB}$ and $6.5 \mathrm{~dB}$ difference between 4-QAM and 16-QAM, and 16-QAM and 64-QAM.

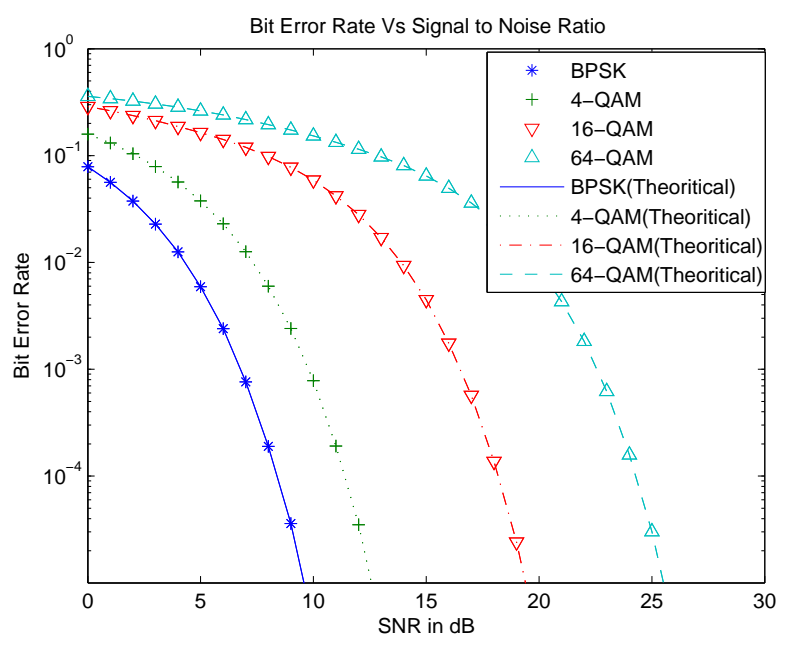

Fig. 1. Stylized BER versus SNR Performance Curves

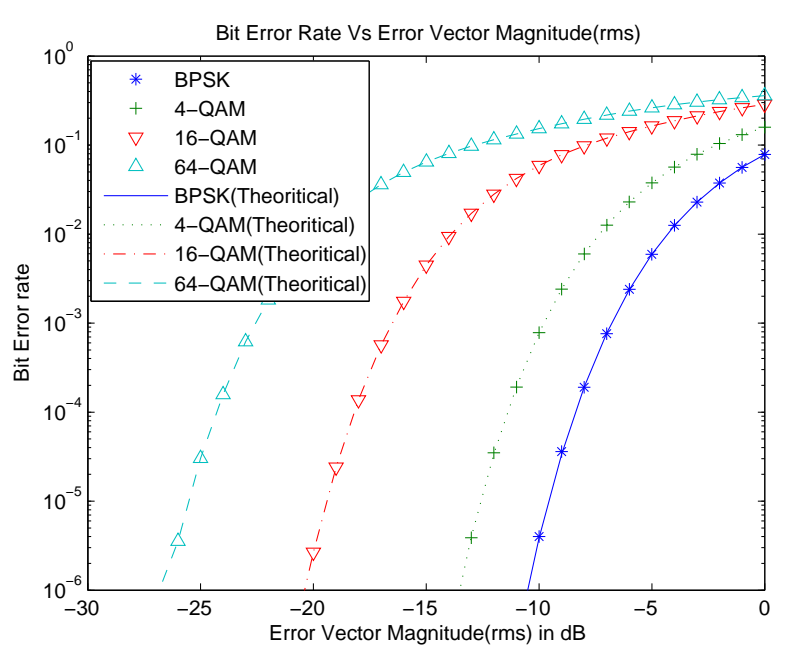

Fig. 2. Stylized BER versus EVM Performance Curves

\section{CONCLUSIONS}

Extended relationships among the bit error rate, signal to noise ration and error vector magnitude are shown in this paper. Due to normalization, the EVM is the same for a given SNR, and they maintain an inverse relationships between them. Since error vector magnitude can be directly measured from the down converted signals using vector signal analyzers, it can save the extra calculations that may be required to find out the bit error rates, which is more of a end to end comparison. In many adaptive systems, this can also simplify the cost function calculation greatly. However, for large streams or packets the EVM calculation may be expensive. Effect of 


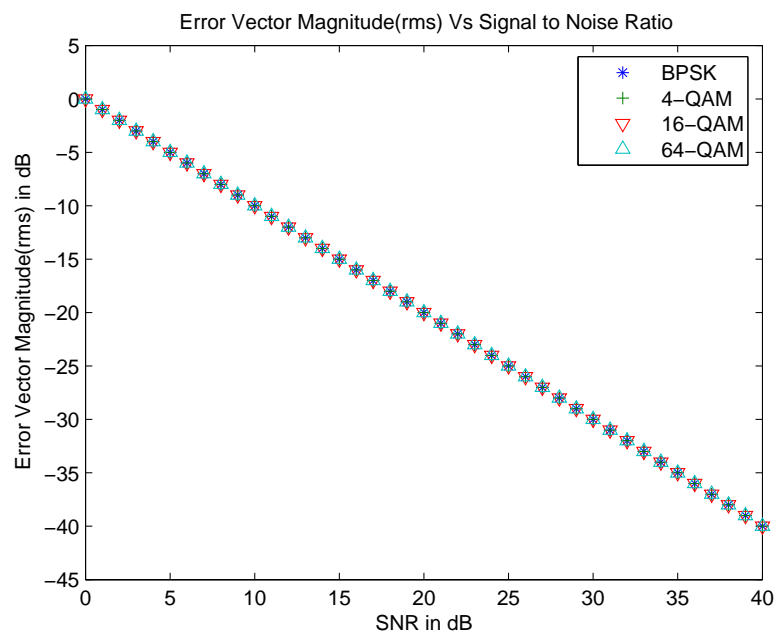

Fig. 3. Stylized EVM versus BER Performance Curves

different fading environments, effect of using EVM-adaptive $M$-ary modulation systems instead of BER-adaptive systems are now being considered as an extension of the work.

\section{ACKNOWLEDGMENT}

The authors would like to thank Mr. Nabil Shovon Ashraf for his generous assistance throughout the work.

\section{REFERENCES}

[1] S. Forestier, P. Bouysse, R. Quere, A. Mallet, J. Nebus, and L. Lapierre. "Joint optimization of the power-aided efficiency and error vector measurement of 20-GHz pHEMT amplifier through a new dynamic biascontrol method". IEEE Transactions on Microwave Theory and Techniques, vol.52(no.4):pp.1132-1140, Apr. 2004.

[2] K. Ghairabeh, K. Gard, and M. Steer. "Accurate Estimation of Digital Communication System Metrices - SNR, EVM and $\rho$ in a Nonlinear Amplifier Environment". IEEE Transactions on Communications, pages pp.734-739, Sept. 2005.

[3] A. Goldsmith. Wireless Communications. Cambridge University Press, Stanford University, 1st edition edition, 2005.

[4] L. Hanzo, W. Webb, and T. Keller. Single-and Multi-Carrier Quadrature Amplitude Modulation. Wiley, Chichester, 2nd edition, 2000.

[5] IEEE, IEEE Standard 802.11b-1999. IEEE Standard for Wireless LAN Medium Access Control (MAC) and Physical Layer (PHY) Specifications: High Speed Physical Layer Extension in the $2.4 \mathrm{GHz}$ Band.

[6] IEEE, IEEE Standard 802.11a-1999. IEEE Standard for Wireless LAN Medium Access Control (MAC) and Physical Layer (PHY) Specifications: High Speed Physical Layer in the $5 \mathrm{GHz}$ Band.

[7] M. D. McKinley, K. A. Remley, M. Mylinski, J. S. Kenney, D. Schreurs, and B. Nauwelaers. "EVM Calculation for Broadband Modulated Signals". Technical Report, 2005. Work of United States Goverment

[8] S. Yi, C. Tsimenidis, O. Hinton, and B. Sharif. "Adaptive minimum bit error rate multiuser detection for asynchronous MC-CDMA systems frequency selective Rayleigh fading channels". 14th IEEE 2003 International Symposium on Personal, Indoor and Mobile Radio Communications. Proceedings (IEEE Cat. No.03TH8677)., vol.2:pp.902906, Apr. 2004. 